\title{
Relación entre el puntaje de ingreso y el rendimiento académico de los estudiantes de la Facultad de Ciencias Matemáticas de la UNMSM que ingresaron mediante los exámenes de admisión 2010-2013.
}

\section{Martha Olinda Gonzales Bohorquez ${ }^{1}$}

Resumen: El presente trabajo de investigación estudia la relación entre el puntaje de ingreso y el coeficiente de rendimiento académico (CRA), de una población conformada por los estudiantes de la Facultad de Ciencias Matemáticas que ingresaron mediante los exámenes de admisión 2010-2013 de la UNMSM. Por medio del CRA se clasificó según rendimiento académico a la población en estudio, evidenciándose que más del $80 \%$ de la población clasifica como estudiante en riesgo académico. Para cada estrato de la población se elaboraron tablas de contingencia y se utilizó el coeficiente de correlación de Pearson para estudiar la relación entre el puntaje de ingreso y el CRA. Los resultados obtenidos evidenciaron que los que ingresaron con los puntajes más altos a la FCM no son necesariamente los que tienen mejor rendimiento académico en los dos primeros años de estudio, pero sí los que tienen más posibilidades de clasificar como estudiante de rendimiento medio o superior.

Palabras clave: puntaje de ingreso; coeficiente de rendimiento académico; estudiante en riesgo académico; estudio descriptivo correlacional.

Relationship between the entrance score and the academic performance of the students of the Faculty of Mathematical Sciences of the UNMSM who entered through the admission exams 2010-2013.

\begin{abstract}
The present study examines the relationship between the entrance examination score and the academic performance coefficient (CRA) of a population made up of the students of the Faculty of Mathematical Sciences who through the admission exams 2010 - 2013 of the UNMSM were admitted in the FCM. Through the CRA, the study population was classified according to academic performance, showing that more than $80 \%$ of the population classifies as a student at academic risk. For each stratum of the population, contingency tables were prepared and the Pearson correlation coefficient was used to study the relationship between the income score and the ARC. The results obtained showed that those who entered with the highest scores to the FCM are not necessarily those who have better academic performance in the first two years of study, but those who are more likely to classify as a student with average or higher performance.
\end{abstract}

Keywords: entrance examination score; academic performance coefficient; student at academic risk; descriptive and correlational Study.

Recibido: 09/11/2017. Aceptado: 26/12/2017. Publicado online: 31/12/2017

(C)Los autores. Este artículo es publicado por la Revista PESQUIMAT de la Facultad de Ciencias Matemáticas, Universidad Nacional Mayor de San Marcos. Este es un artículo de acceso abierto, distribuido bajo los términos de la licencia Creative Commons Atribucion-No yor de Sa Comercia-Compartir Igual 4.0 Internacional.(http://creativecommons.org/licenses/by-nc-sa/4.0/) que permite el uso no comercial, distribu-
ción y reproducción en cualquier medio, siempre que la obra original sea debidamente citada. Para información, por favor póngase en contacto con revistapesquimat.matematica@unmsm.edu.pe

\footnotetext{
${ }^{1}$ UNMSM, Facultad de Ciencias Matemáticas, e-mail: mgonzalesb@unmsm.edu.pe
} 


\section{Introducción}

La UNMSM tiene establecido un proceso de admisión que ejecuta cada año a través de la Oficina Central de Admisión (OCA), durante las últimas décadas, desde 1970; la UNMSM ha mantenido con ligeras modificaciones un modelo de examen de admisión consistente en un examen objetivo de selección múltiple, con la única excepción del año 2006 en la que optó por un nuevo modelo que constó de una primera prueba objetiva de carácter eliminatorio y una segunda prueba desarrollada. Estos exámenes han variado en el número de sus preguntas y/o en la distribución de las mismas.

Se sabe que la oficina central de admisión de la UNMSM a través de sus dependencias realiza diversos estudios sobre el examen general de admisión con respecto a su capacidad técnica, correcta estructuración y adecuación con los estándares de calidad, a fin de mejorar la calidad de los procesos de selección académica para el ingreso a la vida universitaria.

Según Miljánovich M. (2005) [2], una de las condiciones para que un modelo de examen de admisión a la universidad se considere adecuado es que los puntajes de ingreso de los estudiantes deben correlacionarse positiva y significativamente con los rendimientos académicos en los estudios universitarios.

Según Ocaña F Y.(2011) [3], "en nuestra universidad (UNMSM), aún no se ha realizado un estudio de este tipo, que busque encontrar la correlación entre los resultados del examen de admisión y el rendimiento académico en la universidad". A la fecha no se tiene conocimiento de un trabajo concluido y publicado del tema que es motivo del presente estudio de investigación, por ello considero importante explorar e investigar respecto de la relación entre el puntaje de ingreso y el rendimiento académico de los estudiantes de la FCM que ingresaron mediante los exámenes de admisión 2010-2013 de la UNMSM y así en base a los resultados tener un panorama más amplio sobre el cual se pueda evaluar la pertinencia de establecer nuevos criterios para la continua mejora de los instrumentos de selección de esta casa de estudio, así como evaluar la pertinencia de diseñar nuevas estrategias que brinden el apoyo necesario a los estudiantes de la FCM para mejorar su rendimiento académico. Este trabajo de investigación se enmarca dentro de este objetivo.

\section{Metodología}

La investigación es de tipo descriptivo correlacional y el trabajo se realizó en base a la información proporcionada por la Dirección Académica de la FCM y la Oficina Central de Admisión de la UNMSM con los que se elaboró una base de datos de la población en estudio, la cual está dividida en cuatro estratos según año de ingreso (2010, 2011,2012 y 2013). Esta base de datos fué procesada adecuadamente, para el logro de los siguientes objetivos: Clasificar cada estrato de la población según rendimiento académico a través del coeficiente de rendimiento académico (CRA) y Medir el grado de relación entre el puntaje de ingreso y el CRA en cada estrato.

\subsection{Procedimiento de recolección de datos}

Para la recolección de datos se cursaron las siguientes solicitudes

Se solicitó a la Dirección Académica de la Facultad de Ciencias Matemáticas, la información referente a los datos académicos de los estudiantes que ingresaron por medio del examen general de admisión 2010 - 2013.

Se solicitó a la señora Decana de la Facultad de ciencias Matemáticas, una carta de presentación dirigida al Director de la Oficina Central de Admisión de la UNMSM. 
Se solicitó a la Oficina Central de Admisión de la UNMSM la información referente a los puntajes de ingreso obtenidos por los ingresantes a la FCM en los exámenes de admisión 2010 - 2013. Se adjuntó a la solicitud, la carta de presentación expedida por la Decana de la FCM.

Se organizó una base de datos con la información proporcionada referente a los datos académicos de los estudiantes y al puntaje de ingreso que obtuvieron en el examen de admisión.

\subsection{Descripción de la población estudiada}

La población estudiada estuvo conformada por los estudiantes de la Facultad de Ciencias Matemáticas que ingresaron mediante los exámenes de admisión 2010 - 2013 de la UNMSM.

Dicha población se dividió en cuatro estratos, según año de ingreso. Por ejemplo el primer estrato corresponde al total de ingresantes mediante el examen de admisión 2010 matriculados en alguna de las cuatro escuelas académico profesionales de la Facultad de Ciencias Matemáticas de la UNMSM, que según la siguiente tabla 1 suman 263 estudiantes.

\begin{tabular}{ccccc}
\hline \multicolumn{5}{c}{ Tabla 1. Facultad de Ciencias Matemáticas - UNMSM } \\
Población en Estudio por Año de Ingreso y Escuela Académico Profesional \\
\hline Escuela & $\mathrm{N}^{\circ}$ & $\mathrm{N}^{\circ}$ & $\mathrm{N}^{\circ}$ & $\mathrm{N}^{\circ}$ \\
Académico & de estudiantes & de estudiantes & de estudiantes & de estudiantes \\
Profesional & $\begin{array}{c}\text { Ingresantes por } \\
\text { examen de }\end{array}$ & $\begin{array}{c}\text { Ingresantes por } \\
\text { examen de }\end{array}$ & $\begin{array}{c}\text { Ingresantes por } \\
\text { examen de }\end{array}$ & $\begin{array}{c}\text { Ingresantes por } \\
\text { examen de }\end{array}$ \\
& admisión 2010 & admisión 2011 & admisión 2012 & admisión 2013 \\
Matemática & 79 & 86 & 75 & 97 \\
Estadística & 49 & 53 & 63 & 32 \\
Inv. Operativa & 77 & 89 & 101 & 87 \\
Computación Científica & 58 & 58 & 67 & 63 \\
\hline Total & 263 & 286 & 306 & 279 \\
\hline
\end{tabular}

\subsection{Definiciones de términos básicos relativos al estudio}

Puntaje de ingreso: Se refiere al puntaje obtenido en el examen de admisión por los ingresantes a las distintas Escuelas Académico Profesionales de la Facultad de Ciencias Matemáticas de la Universidad Nacional Mayor de San Marcos.

Rendimiento académico: El rendimiento académico es un constructo susceptible de adoptar valores cuantitativos y cualitativos, a través de los cuales existe una aproximación a la evidencia y dimensión del perfil de habilidades, conocimientos, actitudes y valores desarrollados por el estudiante en el proceso de enseñanza aprendizaje [1].

Estudiante en Riesgo escolar o Académico: Se identifica con aquel estudiante que tiene problemas de reprobación, bajo aprovechamiento y/o deserción [7].

Coeficiente de rendimiento académico (CRA): Es un factor que clasifica a los estudiantes por rendimiento académico y se determina mediante la siguiente ecuación [4]:

$$
C R A=P R\left(\frac{C H A}{C H M}\right)\left(\frac{C H A}{S}\right)
$$


Donde

$P R=$ Promedio general

$C H M=$ Créditos históricos matriculados

$C H A=$ Créditos históricos aprobados

$S=$ Número de semestres.

Considerando en promedio 22 créditos por semestre, en cuatro semestres el máximo obtenible del CRA es 440. Los estudiantes se clasifican en cuatro grupos.

\begin{tabular}{lc}
\hline CLASIFICACIÓN & CRA \\
\hline Estudiante en riesgo académico & $(0,100]$ \\
Estudiante de rendimiento inferior & $(100,200]$ \\
Estudiante de rendimiento medio & $(200,300]$ \\
Estudiante de rendimiento superior & $(300,440]$
\end{tabular}

\section{Tratamiento Estadístico}

Con la información recopilada referente a los datos académicos de los estudiantes que ingresaron por medio del examen de admisión 2010 - 2013, se procedió a calcular el PR,CHM,CHA,S y el coeficiente de rendimiento académico (CRA) de cada uno de ellos, mediante el uso del software Microsoft Office Excel 2010.

Mediante el software SPSS versión 20 se procesó la base de datos de cada estrato de la población, para:

- Realizar la clasificación de los estudiantes por rendimiento académico a través del CRA, tomando como referencia los dos primeros años de estudio.

- Obtener las tablas de contingencia del puntaje de ingreso vs el CRA [5].

- Correlacionar el puntaje de ingreso y el CRA de los estudiantes mediante el coeficiente de correlación de Pearson.

\section{Resultados y Discusión}

\subsection{Clasificación de la población en estudio según rendimiento académico}

Los resultados de la clasificación de cada estrato de la población en estudio según rendimiento académico, se muestran en las siguientes tablas conjuntamente con las correspondientes tablas de contingencia que nos permiten analizar la asociación de los puntajes de ingreso y el rendimiento académico. 
Tabla 2. Clasificación de estudiantes por Rendimiento Académico en los dos primeros años de estudio. FCM - Ingresantes 2010

\begin{tabular}{lcc}
\hline \multicolumn{1}{c}{ Clasificación } & Frecuencia & Porcentaje válido \\
\hline Estudiante en riesgo académico & 212 & 80,6 \\
Estudiante de rendimiento inferior & 41 & 15,6 \\
Estudiante de rendimiento medio & 8 & 3,0 \\
Estudiante de rendimiento superior & 2 & 0,8 \\
\hline Total & 263 & 100,0
\end{tabular}

Tabla 3. Puntaje de Ingreso vs CRA

FCM - Ingresantes por Examen de admisión 2010

\begin{tabular}{|l|c|c|c|c|c|}
\hline \multirow{2}{*}{$\begin{array}{c}\text { Puntaje de } \\
\text { ingreso } \\
\text { (cuartiles) }\end{array}$} & \multicolumn{3}{|c|}{ Clasificación según rendimiento académico } & \\
\cline { 2 - 5 } & $\begin{array}{c}\text { Estudiante en } \\
\text { riesgo } \\
\text { académico }\end{array}$ & $\begin{array}{c}\text { Estudiante de } \\
\text { rendimiento } \\
\text { inferior }\end{array}$ & $\begin{array}{c}\text { Estudiante de } \\
\text { rendimiento } \\
\text { medio }\end{array}$ & $\begin{array}{c}\text { Estudiante de } \\
\text { rendimiento } \\
\text { superior }\end{array}$ & Total \\
\hline puntaje muy bajo & 62 & 4 & 0 & 0 & 66 \\
puntaje bajo & 58 & 8 & 0 & 0 & 66 \\
puntaje medio & 46 & 17 & 2 & 1 & 66 \\
puntaje alto & 46 & 12 & 6 & 1 & 65 \\
\hline \multicolumn{1}{|c|}{ Total } & 212 & 41 & 8 & 2 & 263 \\
\hline
\end{tabular}

Análisis de la tabla 3

1) Se observa que 212 estudiantes, que equivale al $80,6 \%$ del total de estudiantes; está en situación de riesgo académico y 10 estudiantes que equivale al 3,8 \% del total de estudiantes, clasifica como estudiante de rendimiento medio o estudiante de rendimiento superior. Cabe resaltar que ese 3,8\% sólo está compuesto por estudiantes que ingresaron con puntaje medio o puntaje alto.

2) De los estudiantes que ingresaron con puntaje muy bajo o bajo, ninguno logró clasificar como estudiante de rendimiento medio o superior, la gran mayoría de ellos, el 90,9\%; clasificó como estudiante en riesgo académico.

3) De los 65 estudiantes que ingresaron con puntaje alto, el $70 \%$ (46) clasificaron como estudiante en riesgo académico.

Conclusión: De 1 y 2 podemos concluir que los estudiantes que ingresaron con puntaje medio o alto son los que tiene más posibilidades de clasificar como estudiantes de rendimiento medio o superior.

De 3 se concluye que no necesariamente los que ingresaron con puntaje alto clasificaron como estudiante de rendimiento medio o superior. 
Tabla 4. Clasificación de estudiantes por Rendimiento Académico en los dos primeros años de estudio. FCM - Ingresantes 2011

\begin{tabular}{lcc}
\hline \multicolumn{1}{c}{ Clasificación } & Frecuencia & Porcentaje válido \\
\hline Estudiante en riesgo académico & 252 & 88,11 \\
Estudiante de rendimiento inferior & 25 & 8,74 \\
Estudiante de rendimiento medio & 9 & 3,15 \\
Estudiante de rendimiento superior & 0 & 0,0 \\
\hline Total & 286 & 100,0 \\
\hline
\end{tabular}

Tabla 5. Puntaje de Ingreso vs CRA

FCM - Ingresantes por Examen de admisión 2011

\begin{tabular}{|l|c|c|c|c|c|}
\hline \multirow{2}{*}{$\begin{array}{c}\text { Puntaje de } \\
\text { ingreso } \\
\text { (cuartiles) }\end{array}$} & \multicolumn{3}{|c|}{ Clasificación según rendimiento académico } & \\
\cline { 2 - 5 } & $\begin{array}{c}\text { Estudiante en } \\
\text { riesgo } \\
\text { académico }\end{array}$ & $\begin{array}{c}\text { Estudiante de } \\
\text { rendimiento } \\
\text { inferior }\end{array}$ & $\begin{array}{c}\text { Estudiante de } \\
\text { rendimiento } \\
\text { medio }\end{array}$ & $\begin{array}{c}\text { Estudiante de } \\
\text { rendimiento } \\
\text { superior }\end{array}$ & Total \\
\hline puntaje muy bajo & 68 & 2 & 1 & 0 & 71 \\
puntaje bajo & 67 & 5 & 0 & 0 & 72 \\
puntaje medio & 59 & 9 & 4 & 0 & 72 \\
puntaje alto & 58 & 9 & 4 & 0 & 71 \\
\hline \multicolumn{1}{|c|}{ Total } & 252 & 25 & 9 & 0 & 286 \\
\hline
\end{tabular}

Análisis de la tabla 5

1) De un total de 286 estudiantes el $88,11 \%$ (252) clasificaron como estudiantes en riesgo académico y ningún estudiante alcanzó un rendimiento superior. Se observa que solo el $3,15 \%$ (9) clasificaron como estudiante de rendimiento medio y que en su mayoría este $3,15 \%$ está compuesto por estudiantes que ingresaron con puntaje medio o puntaje alto.

2) De los estudiantes que ingresaron con puntaje muy bajo o bajo, sólo 1 logró clasificar como estudiante de rendimiento medio, la gran mayoría de ellos, el 94,4\% clasificó como estudiante en riesgo académico.

3) De los 71 estudiantes que ingresaron con puntaje alto el $81,7 \%$ (58) clasificaron como estudiantes en riesgo académico.

Conclusión: De 1 y 2 los estudiantes que ingresaron con puntaje medio o alto son los que tiene más posibilidades de clasificar como estudiantes de rendimiento medio. De 3 podemos concluir que a mayor puntaje de ingreso no necesariamente mejor rendimiento académico. 
Tabla 6. Clasificación de estudiantes por Rendimiento Académico en los dos primeros años de estudio. FCM - Ingresantes 2012

\begin{tabular}{lcc}
\hline \multicolumn{1}{c}{ Clasificación } & Frecuencia & Porcentaje válido \\
\hline Estudiante en riesgo académico & 269 & 87,9 \\
Estudiante de rendimiento inferior & 33 & 10,8 \\
Estudiante de rendimiento medio & 3 & 1,0 \\
Estudiante de rendimiento superior & 1 & 0,3 \\
\hline Total & 306 & 100,0
\end{tabular}

Tabla 7. Puntaje de Ingreso vs CRA

FCM - Ingresantes por Examen de admisión 2012

\begin{tabular}{|l|c|c|c|c|c|}
\hline \multirow{2}{*}{$\begin{array}{c}\text { Puntaje de } \\
\text { ingreso } \\
\text { (cuartiles) }\end{array}$} & \multicolumn{3}{|c|}{ Clasificación según rendimiento académico } & \\
\cline { 2 - 5 } & $\begin{array}{c}\text { Estudiante en } \\
\text { riesgo } \\
\text { académico }\end{array}$ & $\begin{array}{c}\text { Estudiante de } \\
\text { rendimiento } \\
\text { inferior }\end{array}$ & $\begin{array}{c}\text { Estudiante de } \\
\text { rendimiento } \\
\text { medio }\end{array}$ & $\begin{array}{c}\text { Estudiante de } \\
\text { rendimiento } \\
\text { superior }\end{array}$ & Total \\
\hline puntaje muy bajo & 68 & 9 & 0 & 0 & 77 \\
puntaje bajo & 64 & 10 & 2 & 1 & 77 \\
puntaje medio & 65 & 11 & 0 & 0 & 76 \\
puntaje alto & 72 & 3 & 1 & 0 & 76 \\
\hline \multicolumn{1}{|c|}{ Total } & 269 & 33 & 3 & 1 & 306 \\
\hline
\end{tabular}

Análisis de la tabla 7

1) De un total de 306 estudiantes el $87,9 \%$ (269) clasificaron como estudiantes en riesgo académico y sólo el 1,3\% (4) alcanzó un rendimiento medio o superior. Se observa que de ese $1,3 \%$ en su mayoría ( 3 de 4 ) está compuesto por estudiantes que ingresaron con puntaje bajo.

2) De los estudiantes que ingresaron con puntaje muy bajo ninguno logró clasificar como estudiante de rendimiento medio o superior, al igual que los que ingresaron con puntaje medio.

3) De los estudiantes que ingresaron con puntaje muy bajo o bajo, en su mayoría el 85,7\% clasificó como estudiante en riesgo académico, siendo superado este porcentaje con un $94,7 \%$ por los que ingresaron con puntaje alto y que también clasificaron como estudiante en riesgo académico.

Conclusión: Para este estrato de la población no existe relación entre el puntaje de ingreso y el CRA. 
Tabla 8. Clasificación de estudiantes por Rendimiento Académico en los dos primeros años de estudio. FCM - Ingresantes 2013

\begin{tabular}{ccc}
\hline Clasificación & Frecuencia & Porcentaje válido \\
\hline Estudiante en riesgo académico & 247 & 88,5 \\
Estudiante de rendimiento inferior & 26 & 9,3 \\
Estudiante de rendimiento medio & 5 & 1,8 \\
Estudiante de rendimiento superior & 1 & 0,4 \\
\hline Total & 279 & 100,0 \\
\hline
\end{tabular}

Tabla 9. Puntaje de Ingreso vs CRA

FCM - Ingresantes por Examen de admisión 2013

\begin{tabular}{|l|c|c|c|c|c|}
\hline \multirow{2}{*}{$\begin{array}{c}\text { Puntaje de } \\
\text { ingreso } \\
\text { (cuartiles) }\end{array}$} & \multicolumn{3}{|c|}{ Clasificación según rendimiento académico } & \\
\cline { 2 - 5 } & $\begin{array}{c}\text { Estudiante en } \\
\text { riesgo } \\
\text { académico }\end{array}$ & $\begin{array}{c}\text { Estudiante de } \\
\text { rendimiento } \\
\text { inferior }\end{array}$ & $\begin{array}{c}\text { Estudiante de } \\
\text { rendimiento } \\
\text { medio }\end{array}$ & $\begin{array}{c}\text { Estudiante de } \\
\text { rendimiento } \\
\text { superior }\end{array}$ & Total \\
\hline puntaje muy bajo & 66 & 4 & 0 & 0 & 70 \\
puntaje bajo & 67 & 3 & 0 & 0 & 70 \\
puntaje medio & 59 & 9 & 2 & 0 & 70 \\
puntaje alto & 55 & 10 & 3 & 1 & 69 \\
\hline \multicolumn{1}{|c|}{ Total } & 247 & 26 & 5 & 1 & 279 \\
\hline
\end{tabular}

Análisis de la tabla 9

1) De un total de 279 estudiantes el $88,5 \%$ (247) clasificaron como estudiante en riesgo académico y sólo el 2,2\% (6) logró un rendimiento medio o superior, cabe resaltar que este pequeño porcentaje está compuesto solo por estudiantes que ingresaron con puntaje medio o alto.

2) De los estudiantes que ingresaron con puntaje muy bajo o puntaje bajo ninguno clasificó como estudiante de rendimiento medio o superior y el $95 \%$ de este grupo clasificó como estudiante en riesgo académico.

3) De los 69 estudiantes que ingresaron con puntaje alto el 79,7\% (55) clasificaron como estudiante en riesgo académico.

Conclusión: De 1 y 2 podemos concluir que los estudiantes que ingresaron con puntaje medio o alto son los que tiene más posibilidades de clasificar como estudiantes de rendimiento medio o rendimiento superior. De 3 podemos concluir que a mayor puntaje de ingreso no necesariamente mejor rendimiento académico.

Cabe resaltar que en cada uno de los cuatro estratos de la población más del $80 \%$ de estudiantes clasifica como estudiante en riesgo académico y no más del $0,8 \%$ como estudiante de rendimiento superior (ver tablas 2,4,6 y 8 ). 


\subsection{Correlación entre el Puntaje de Ingreso y Coeficiente de Rendimiento Académico}

A continuación se muestran los resultados del estudio de correlación realizado mediante el coeficiente de correlación de Pearson entre el puntaje de ingreso y el CRA en cada estrato de la población, seguido del análisis respectivo.

Tabla 10. Correlación entre el Puntaje de Ingreso y Coeficiente de rendimiento Académico. FCM-Ingresantes 2010.

\begin{tabular}{lll}
\hline Puntaje de ingreso & & \\
& Correlación de Pearson & 0,278 \\
Coeficiente de rendimiento académico & & \\
& Sig. (bilateral) & 0,000 \\
& $\mathrm{~N}$ & 263 \\
\hline
\end{tabular}

De la tabla 10 se observa que el coeficiente de correlación entre el puntaje de ingreso y el coeficiente de rendimiento académico de los estudiantes ingresantes a la FCM por medio del examen de admisión 2010 es $r=0,278$ que indica la existencia de una correlación positiva y débil.

Como $r^{2}=0,077284$, entonces el 7,72\% de la variación en el rendimiento académico de los estudiantes se explica por el puntaje de ingreso obtenido en el examen de admisión, lo cual dice que existen otros factores que explican el $92,28 \%$ de la variación en el rendimiento académico de los estudiantes.

Tabla 11. Correlación entre el Puntaje de Ingreso y Coeficiente de rendimiento Académico. FCM-Ingresantes 2011.

\begin{tabular}{lll}
\hline Puntaje de ingreso & & \\
& Correlación de Pearson & 0,202 \\
Coeficiente de rendimiento académico & & \\
& Sig. (bilateral) & 0,001 \\
& $\mathrm{~N}$ & 286 \\
\hline
\end{tabular}

De la tabla 11 se observa que el coeficiente de correlación entre el puntaje de ingreso y el coeficiente de rendimiento académico de los estudiantes ingresantes a la FCM por medio del examen de admisión 2011 es $r=0,202$, lo cual indica que existe una correlación positiva y débil. Como $r^{2}=0,040804$, entonces $4,08 \%$ de la variación en el rendimiento académico de los estudiantes se explica por el puntaje de ingreso obtenido en el examen de admisión, y un 95,92\% de la variación en el rendimiento académico se explican por otros factores. 
Tabla 12. Correlación entre el Puntaje de Ingreso y Coeficiente de rendimiento Académico. FCM-Ingresantes 2012.

\begin{tabular}{lll}
\hline Puntaje de ingreso & & \\
& Correlación de Pearson & 0,031 \\
Coeficiente de rendimiento académico & & \\
& Sig. (bilateral) & 0,590 \\
& $\mathrm{~N}$ & 306 \\
\hline
\end{tabular}

De la tabla 12 se observa que el coeficiente de correlación entre el puntaje de ingreso y el coeficiente de rendimiento académico de los estudiantes ingresantes a la FCM por medio del examen de admisión 2012 es $r=0,031$, lo cual indica una correlación muy débil casi nula. En este estrato de la población no hay relación entre el puntaje de ingreso y el rendimiento académico.

Tabla 13. Correlación entre el Puntaje de Ingreso y Coeficiente de rendimiento Académico. FCM-Ingresantes 2013.

\begin{tabular}{lll}
\hline Puntaje de ingreso & & \\
& Correlación de Pearson & 0,206 \\
Coeficiente de rendimiento académico & & \\
& Sig. (bilateral) & 0,001 \\
& $\mathrm{~N}$ & 279 \\
\hline
\end{tabular}

De la tabla 13 se observa que el coeficiente de correlación entre el puntaje de ingreso y el coeficiente de rendimiento académico de los estudiantes ingresantes a la FCM por medio del examen de admisión 2013 es $r=0,206$, lo cual indica que existe una correlación positiva y débil. Como $r^{2}=0,042436$, entonces $4,24 \%$ de la variación en el rendimiento académico de los estudiantes se explica por el puntaje de ingreso obtenido en el examen de admisión, lo cual manifiesta que un $95,76 \%$ de la variación en el rendimiento académico se explica por otros factores.

\section{Conclusión}

Tomando en cuenta los resultados del análisis estadístico en lo que corresponde a la correlación del puntaje de ingreso y el coeficiente de rendimiento académico de la población estudiada, así como el resultado del análisis de las tablas de contingencia en cada estrato de la población podemos afirmar que los estudiantes que obtienen los más altos puntajes de ingreso a la FCM, de la UNMSM, no necesariamente son los que tienen mejor rendimiento académico en los dos primeros años de estudio, pero sí son los que tienen más posibilidades de clasificar como estudiantes de rendimiento medio o superior. 
En la clasificación según rendimiento académico durante los dos primeros años de estudios, de la población estudiada, se evidenció que más del $80 \%$ de esta población son estudiantes en riesgo académico, es decir son estudiantes que tiene problemas de reprobación, bajo aprovechamiento y/o deserción [7]. Más del 95,6\% de la población estudiada, clasifica como estudiante en riesgo académico o de rendimiento inferior. El porcentaje de estudiantes de rendimiento medio, en ninguno de los cuatro estratos de la población estudiada, supera el $4 \%$ y el porcentaje de estudiantes de rendimiento superior no llega al 1\%. Es importante resaltar que los resultados obtenidos con respecto al rendimiento académico en cada estrato de la población son muy similares.

\section{Recomendaciones}

1. Para los estudiantes en riesgo académico y los de bajo rendimiento, los mismos que deberán detectarse desde los primeros semestres, se recomienda, diseñar nuevas estrategias y fortalecer las ya existentes a fin de poderles brindar el apoyo necesario para mejorar su rendimiento académico [6].

2. Aunque que existen otras variables o características propias de los estudiantes que influyen en el rendimiento académico, es necesario continuar desplegando esfuerzos a fin de que se siga mejorando el instrumento de selección para el ingreso a esta casa de estudios.

3. Los resultados acerca de la clasificación de los estudiantes de la FCM según su rendimiento académico son muy similares y no gratos, es menester realizar estudios periódicos de seguimiento en el rendimiento académico de los estudiantes, con el fin de verificar, si los correctivos y/o estrategias diseñadas y aplicadas dan los cambios y resultados esperados.

4. Realizar estudios de investigación que involucren otros factores que influyen en el rendimiento académico de los estudiantes de la FCM.

\section{Agradecimiento}

Expreso mi sincero agradecimiento a la Facultad de Ciencias Matemáticas y a la Oficina Central de Admisión de la UNMSM por brindarme su apoyo proporcionándome la información necesaria para el estudio realizado.

\section{Referencias bibliográficas}

[1] Edel, R. (2003) El rendimiento académico: concepto, investigación y desarrollo. Revista Electrónica Iberoamericana sobre Calidad, Eficacia y Cambio en Educación, 1 (2),1 - 16.

[2] Mijánovich, M.(2005). Fundamentación del nuevo modelo de prueba de admisión de la Universidad Nacional Mayor de San Marcos. Revista de investigación de la Facultad de Psicología de la UNMSM, 8 (1), 139 - 144.

[3] Ocaã, Y. (2011). Variables académicas que influyen en el rendimiento académico de los estudiantes universitarios. Revista de investigación Educativa, 15 (27),165 - 179.

[4] Olivera, L. (2012). Docencia Universitaria: Reflexiones y experiencias. Perú: Fondo Editorial Pontificia Universidad Católica del Perú.

[5] Pérez, C. (2009). Técnicas de análisis de datos con SPSS 15. Madrid: Pearson Educación S.A. 
[6] Robles, R. (2005). Orientación Educativa y Rendimiento académico. Revista Mexicana de orientación educativa, 2 (4), 52 - 66.

[7] Uberetagoyena, S. y Galicia, A. (2009). Propuesta metodológica para detección de alumnos en riesgo escolar en licenciatura. México: Instituto Politécnico Nacional, . 